in vivo $35: 3175-3180(2021)$

doi:10.21873/invivo.12612

\title{
Intraluminal Titanium Alloy Stent to Prevent Tracheal Stenosis in Tracheal Anastomosis
}

\author{
TAE-GI LEE ${ }^{1 *}$, SEOK JIN JANG $^{2 *}$ and SEOK HWA CHOI ${ }^{1}$ \\ ${ }^{1}$ College of Veterinary Medicine, Chungbuk National University, Cheongju, Republic of Korea; \\ ${ }^{2}$ Onnuri Animal Medical Center, Cheongju, Republic of Korea
}

\begin{abstract}
Background/Aim: Tracheal stenosis can cause respiratory problems in mature, small-breed dogs. This study aimed to evaluate the placement of an intratracheal titanium alloy stent to prevent tracheal stenosis in canine tracheal anastomosis. Materials and Methods: The self-expandable intratracheal stent was an alloy of nickel and titanium, at the same atomic ratio. Vital signs and respiratory patterns, $C$ reactive protein, radiography, computed tomography, and endoscopy results after intraluminal stenting were assessed for 3-5 months. Results: No dogs showed evidence of intraluminal tracheal stenosis or tracheitis in the region of stent insertion on tracheoscopy and computed tomography after tracheal stent placement. After 1-2 weeks of tracheal stent placement, all dogs resolved coughing and dyspnea signs and resumed normal activities. Conclusion: The intratracheal stent showed no movement or deformation in the trachea, and had flexibility and an appropriate radial force. Therefore, titanium alloy tracheal stents are useful in stenotic operations for tracheal reconstruction.
\end{abstract}

The trachea is the air pathway form the larynx to the lungs. The trachea is a flexible tube with a sturdy C-shaped cartilage ring. Tracheal trauma and stenosis cause significant morbidity and mortality. Acquired tracheal stenosis may result from various disorders, including neoplasia, inflammation, infection, and trauma (1). Tracheal stenosis includes a variety of conditions, including tracheomalacia,

This article is freely accessible online.

*These Authors contributed equality to this work.

Correspondence to: Seok Hwa Choi, DVM, Ph.D., Department of Veterinary Surgery, College of Veterinary Medicine, Chungbuk National University, Chungdae-ro 1, Seowon-Gu, Cheongju, Chungbuk, 28644, Republic of Korea. Tel: +82 432613144, Fax: +82 432613224, e-mail: shchoi@cbnu.ac.kr

Key Words: Tracheal stenosis, titanium alloy, intratracheal stent, dog. dorsal membrane weakness, cartilage ring malformation, or a combination of these, resulting in varying degrees of raspy, honking breathing, along with coughing and dyspnea.

The initial treatment often involves minimization of factors that cause weight loss, decreased activity, anxiety, and agitation. Conservative medical therapies include a combination of medications such as corticosteroids, antitussives, antibiotics, bronchodilators, tranquilizers, sedatives, and antihistamines $(2,3)$.

Canine tracheal stenosis treatment for restoring a stenotic or obstructed lumen includes placement of extra-luminal rings and intraluminal stents (4). Precise tracheal measurements and subsequent stent size decisions likely contribute, at least in part, to minimizing stent-related complications (5). Tracheal stents are self-expanding metallic stents that need to be oversized compared to the normal tracheal diameter to achieve sufficient outward radial force, complete mucosal apposition, and prevention of stent migration (5).

Advantages of stents include the ability to be used in any region of the trachea, short surgical and anesthesia times, and noninvasive placement $(6,7)$, but complications are not uncommon.

Tracheal reconstruction after partial resection of a stenotic lesion or a malignant tumor is a difficult surgical procedure to perform. The trachea is one of the few organs that are exceptionally difficult to transplant because of the technical difficulty of restoring blood supply to the anastomotic line (8). Tracheal stenosis in dogs is a common irreversible and progressive disease of the airways in middle-aged small and toy breed dogs (9). End-to-end anastomotic repair by a simple interrupted suture is the gold standard surgical repair technique after partial tracheal resection (10). Anastomotic complications after tracheal resection are granulation, stenosis, and separation at the anastomotic line (11). The complications can result in severe morbidity and mortality in toy and miniature dogs. Before this study, we thought there would be no significant advancements in the application of tracheal stents if the migration and fracture of the tracheal stent could not be overcome. 
This study was conducted to determine whether a titanium alloy intratracheal stent could achieve outward radial force and prevent stenosis in the tracheal anastomosis region.

\section{Materials and Methods}

Canine patients. Three client-owned dogs were recruited for this study. Two 6-year-old, $3.5 \mathrm{~kg}$ female miniature poodles and a 9year-old, $2.9 \mathrm{~kg}$ neutered male Yorkshire terrier were referred to the Pusan Veterinary Hospital with histories of being no longer responsive to conservative medical therapy.

Physical examination indicated the physiological parameters were normal, but chest auscultation detected stridor, respiratory distress, and loud inspiratory murmur. Thoracic radiographs showed smaller than normal lung volumes and narrow cranio-thoracic tracheal lumens. The lateral cervical trachea showed moderate-tosevere stenosis of the tracheal inner diameter.

Preparation of intratracheal stent. A flexible stent (FAUNA STENT ${ }^{\circledR}$, MI Tech Co., Seoul, Republic of Korea) with a diameter of $10 \mathrm{~mm}$ and a length of $40 \mathrm{~mm}$ was inserted into the tracheal resection site (Figure 1A). The self-expandable intratracheal stent was an alloy of nickel and titanium with the two elements having the same atomic ratio. The stent was handmade using a cross-andhook knitting method. The radial force of the intratracheal stent was $268.4 \pm 4.2 \mathrm{gf}$, and stent wire thickness was $117 \mu \mathrm{m}$. Radiopaque markers were attached to both ends of the stent and above and below the stent to confirm the placement of the stent in the dog's trachea. The diameter of the intratracheal stent selected for each dog was the same size as the inner diameter of the resected trachea measured on the lateral chest radiograph.

Intratracheal stent insertion in dogs. The dogs were administered xylazine (Rompun ${ }^{\circledR}$, Bayer Co., Seoul, Republic of Korea, $2 \mathrm{mg} / \mathrm{kg}$ ) and tiletamine/zolazepam intramuscularly (Zoletil ${ }^{\circledR}$, Virbac Co., Carros, France, $10 \mathrm{mg} / \mathrm{kg}$ ) immediately before the procedure to induce anesthesia and were also given meloxicam $\left(0.2 \mathrm{mg} / \mathrm{kg}\right.$, Metacam ${ }^{\circledR}$, Boehringer Ingelheim, Ingelheim, Germany) subcutaneously for analgesia. Three to five tracheal rings were resected in the damaged tracheal area by incision of the skin on the neck (Figure 1B). Trachelorrhaphy was performed in a simple interrupted suture pattern using an absorbable monofilament suture type (4-0 Maxon $^{\mathrm{TM}}$, COVIDIEN $^{\mathrm{TM}}$, Minneapolis, MN, USA), and the wound was closed in the usual fashion. Tracheal stitching was performed as an end-toend anastomosis. All dogs were carefully monitored to observe breathing patterns and infections for one week.

After surgery, all dogs were transferred to the recovery room and intravenously administrated 5\% dextrose (5\% Dextrose, Dai Han Pharm Co., Seoul, Republic of Korea) and lactated Ringer's solution (Lactated Ringer's Injection, Dai Han Pharm Co.). Animals were monitored at 15-30 min intervals until consciousness was restored and the ability to lift the head was restored. Frequent monitoring was continued until dogs were able to maintain sternal recumbency. Warm lactated Ringer's solution was administered subcutaneously at $40-80 \mathrm{ml} / \mathrm{kg}$ body weight to prevent dehydration. When normal anesthetic recovery was observed, analgesic meloxicam $(0.2 \mathrm{mg} / \mathrm{kg}$, Metacam ${ }^{\circledR}$, Boehringer Ingelheim) was administered subcutaneously and repeated every 6-12 $\mathrm{h}$ as needed. Dogs were orally administered doxycycline hydrate $(5 \mathrm{mg} / \mathrm{kg}$, Young-pung Pharm., Incheon, Korea), prednisolone (1.1 mg/kg, Yuhan Pharm Co., Seoul, Republic of Korea) and theophylline (10 mg/kg, Alovogen Co., Seoul, Republic of Korea) twice daily.

Radiographic, computed tomographic and tracheoscopic evaluations. Radiographs and computed tomography (CT) images of dogs were observed at 1-week intervals for 2-3 weeks after stent insertion (Figure 1C). Tracheoscopic evaluation was performed in dogs for 2-8 weeks after the intratracheal stent placement. Tracheoscopy was undertaken with a 4-mm rigid endoscope (Karl Storz, Tuttlingen, Germany) and a video-endoscopy system consisting of a video tracheoscope (OTVS70 Pro; Olympus Co., Tokyo, Japan) and a video processor coupled to a light source (CLV-S40 Pro; Olympus Co.). The tracheal wall of the intratracheal stent placement was examined, but no biopsy sampling was performed. All dogs were observed by tracheoscopy for 2-4 weeks at intervals of 2 or 3 weeks after stent insertion.

Analysis of serum C-reactive protein. Serum C-reactive protein (CRP) levels in all dogs were assessed 7 days after tracheal stent insertion. CRP levels were measured using a V200 analyzer (BioNote Co., Seoul, Republic of Korea). Serum (5 $\mu$ l) was collected and added to an analytical diluent bottle. The bottle cap was then closed and the bottle was shaken 5-6 times to mix the sample well. The mixed sample was placed in the test hole of the analyzer and the test results were obtained after $5 \mathrm{~min}$.

Statistical analysis. Statistical analyses were performed using SPSS statistical software package version 19.0.1.1 (IBM Corp., Armonk, NY, USA). Data are presented as mean \pm standard deviation (SD) values. Normality and homogeneity of the data were confirmed before performing analysis of variance (ANOVA). Differences among the experimental groups were assessed by one-way ANOVA followed by Duncan's multiple range test. A $p<0.05$ was considered statistically significant.

\section{Results}

Serum CRP level changes. Serum CRP levels increased in all dogs for up to 3 days after tracheal placement but returned to a normal level after 7 days (Figure 2). Common symptoms of inflammation in dogs, fever, tachycardia, hyperventilation, and anorexia were not detected.

Evaluation of radiographs, CT scans, and tracheoscopy images. Lateral cervical radiographs showed that the intratracheal stents were well placed without movement or damage at the insertion site (Figure 3).

After intratracheal stent placement, the dog's tracheal diameter expanded due to self-expandable radial forces. The intratracheal titanium alloy stents were self-expanding and produced a sufficient external radial force to increase the tracheal diameter and prevent stent movement. None of the dogs exhibited tracheal stenosis or damage at the region of intratracheal stent placement on tracheal endoscopy. However, the intraluminal trachea exhibited a small amount of mucus due to anesthesia (Figure 4A). On CT scans, stent fracture and movement, tracheitis, and obstruction of the stent lumen by granulation tissue at the endotracheal stent insertion 


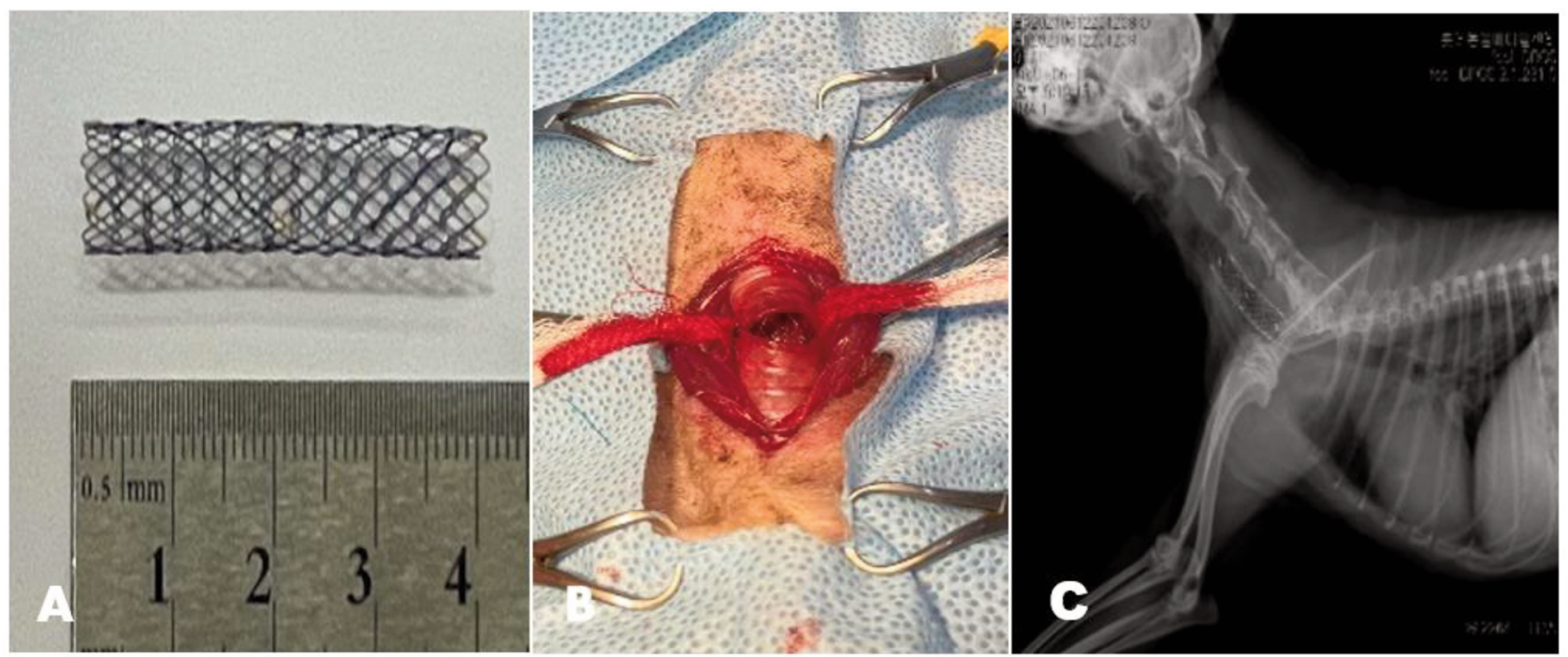

Figure 1. A self-expandable titanium alloy stent (A). Intraoperative image of intratracheal stent insertion after partial tracheal resection (B). A lateral radiograph of an intratracheal stent placement in a dog trachea $(C)$.

\section{C-reactive protein}

250

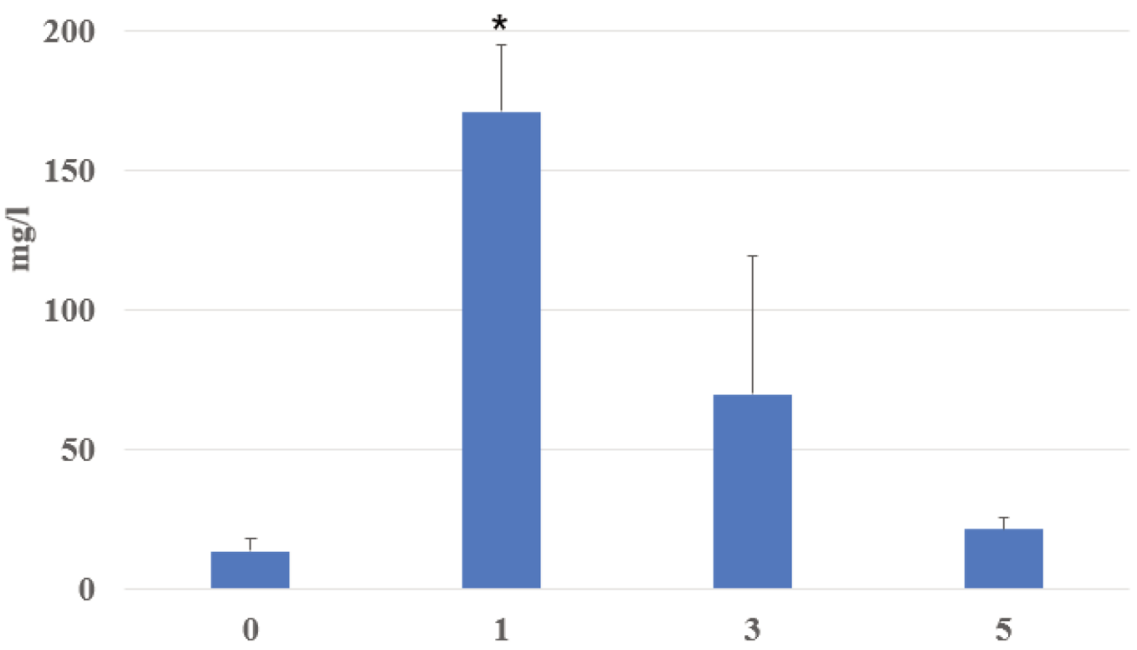

Figure 2. Changes of serum C-reactive protein levels after intratracheal stent placement in dogs. Data are presented as mean $\pm S D$. *Significantly different at $p<0.05$ from day 0 .

site were not observed. Moreover, CT images showed no growth of endothelial cells in the tracheal stent (Figure 4B).

\section{Discussion}

Tracheal cartilage keeps the trachea open, allowing air to enter and leave the lungs. Tracheal stenosis is the result of a multifactorial process producing tracheal cartilage and dorsal membrane weakening and tracheal lumen narrowing (12). Most dogs show immediate clinical improvement after tracheal surgery, but complications have been reported (6), including serious complications such as postoperative laryngeal paralysis and tracheal narrowing (13). Tracheal stenosis can cause serious breathing problems in mature, 

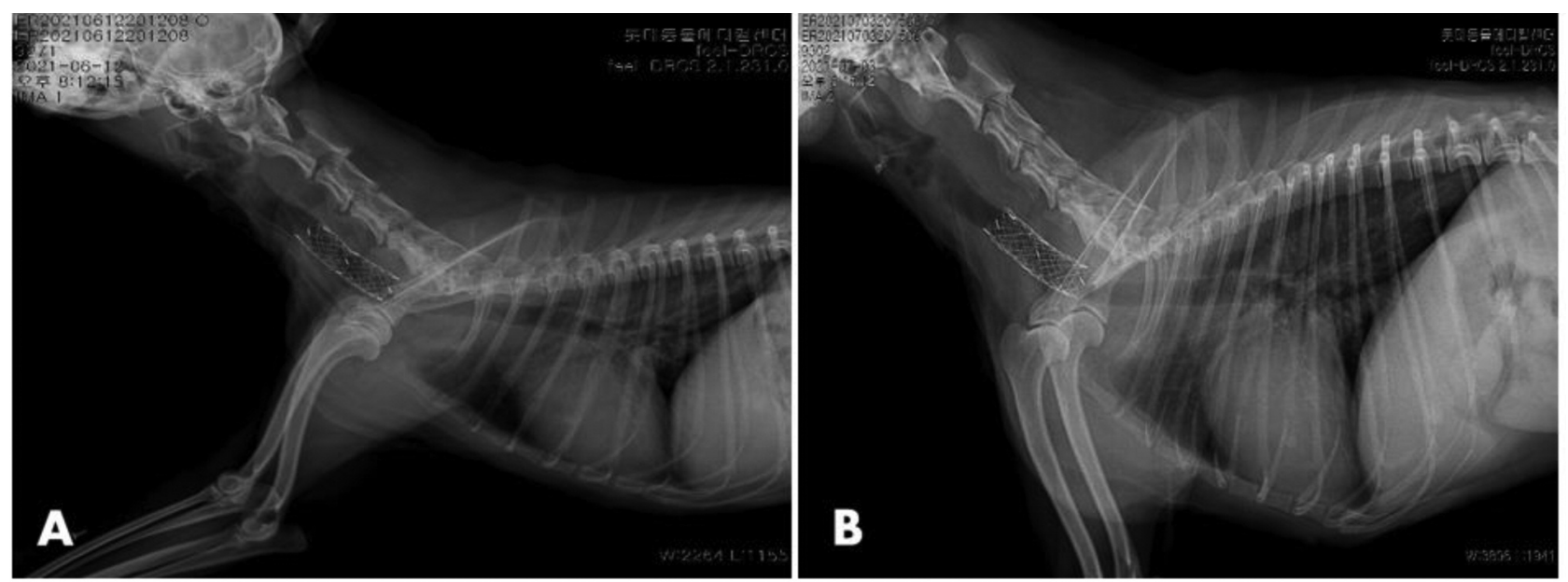

Figure 3. Lateral radiographs 1 day $(A)$ and 21 days $(B)$ after intratracheal stent placement in dogs.

small-breed dogs (14), and in aging dogs (12). The two most common treatments for tracheal stenosis are resection and anastomosis or stenting. The former is technically difficult and can have complications, while the latter requires special equipment and training (15).

Intratracheal stenting helps treat a narrow or obstructed tracheal lumen, and intratracheal stent insertion can be used to treat tracheal stenosis, tumors, and collapse in dogs (16). Dogs with severe tracheal stenosis with symptoms of airway obstruction that are refractory to medical management may be appropriate candidates for tracheal stent insertion (6). Acute complications of tracheal stent insertion are often associated with improper stent sizing or placement, resulting in stent movement or misplacement (13). Several studies have reported intratracheal stent movement and shortening within the trachea $(6,17)$. Dogs with intratracheal stents may be susceptible to chronic and recurrent respiratory tract infections, often developing a need for long-term antibiotic treatment or exhibiting intratracheal granuloma formation. Regardless, intratracheal stenting has become an effective treatment for tracheal stenosis because it is associated with fewer complications than surgery (6).

In this study, radiographs, CT scans, tracheoscopy images, and blood analysis results were obtained to examine stent movements and changes and stent-related tracheal damage. The intratracheal stent used in this study had radiopaque markers attached to both ends of the stent to allow observation of stent's location and shape.

A common sign of tracheal stenosis in the study dogs was dyspnea during inhalation or after exercise. Signs such as coughing, labored breathing, high-pitched and noisy breathing are associated with the presence of tracheal masses (11). Tracheal masses in the study dogs were identified via endoscopy or radiography of the trachea. Histological examination indicated that the intratracheal masses resulted from the presence of foreign objects such as metallic substances or fish bones.

Complications of tracheal stent placement include inflammatory or bacterial tracheitis $(6,18)$, stent fracture $(6$, $18)$, stent migration $(6,19)$, and stent shortening $(19,20)$. If the size of the stent is small, stent movement may occur (5). Therefore, we chose a tracheal stent that was the same size as the patient's tracheal inner diameter. Stent fractures have been reported in $10-42 \%$ of study subjects, depending on the type of stent used (4). Our titanium alloy stents were handmade using a cross-and-hook knitting process, and the two elements had the same atomic ratio. On CT scans, no fractures or deformations of the titanium alloy stents were observed.

Dogs with tracheal stents require regular reevaluation, including chest x-rays and bronchoscopy, as inflammatory or granular tissue has been shown to grow in the lumen of the stent in about one-third of cases (18). Therefore, antitussives, bronchodilators, and anti-inflammatory drugs should be administered after tracheal stenting. A cough caused by the foreign body reaction due to the stent placement can produce marked stress on the tracheal wall. After intratracheal stent placement, our dogs were given corticosteroids, aminophylline, and antibiotics for one week. The dogs were also given analgesics for one week and placed in a quiet environment. We considered it important not to stimulate the dogs for one week during wound healing and stent stabilization after intratracheal stent insertion.

Dogs with tracheal malformations may be at increased risk of developing a thoracic inlet fracture or obstructive tissue ingrowth (21). Our patients showed no tracheal malformation, inflammation at the tracheal connection site, and no penetration of granular tissue into the tracheal stent. The excellent 

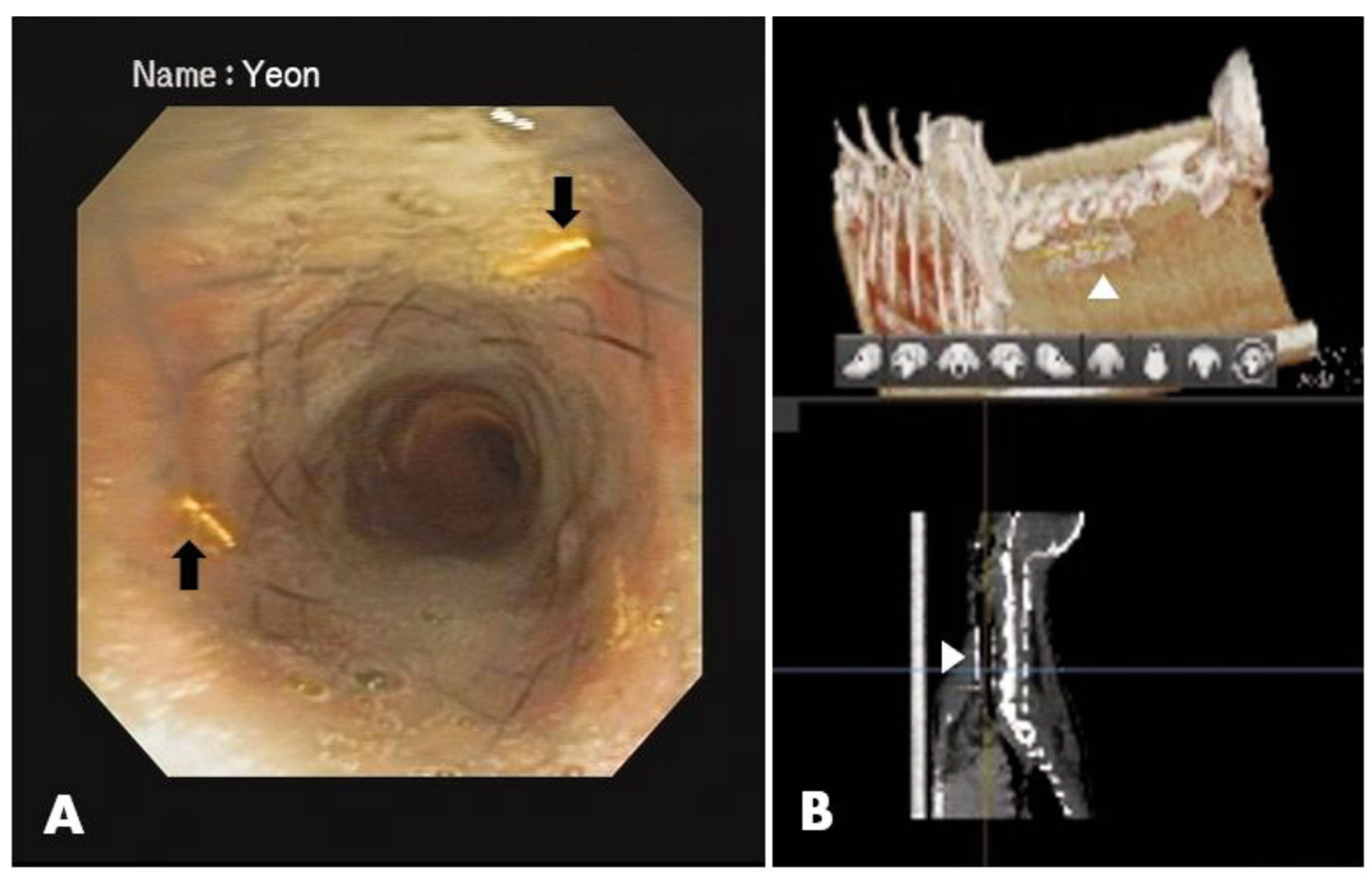

Figure 4. Tracheoscopic image (A) and CT scan (B) 8 weeks after the intratracheal stent placement (arrow heads) in the dog. Radiopaque markers were attached to both ends of the stent and above and below the stent to confirm the placement of the stent in the trachea (arrows).

biological and mechanical properties of titanium alloys have made their use in dental implants very successful (22). We also considered that these properties of titanium would be less irritating in dogs with titanium alloy intratracheal stents.

Serum CRP is an acute-phase protein involved in most forms of inflammation, infection, and tissue necrosis $(23,24)$. CRP level is considered a more reliable parameter than white blood cell counts during acute-phase responses in canine injury (25). In this study, serum CRP was used to assess the degree of intratracheal damage, and the CRP level increased during the 3 days after stent insertion but returned to normal 7 days later. We observed all patients for 3-5 months, but no inflammatory symptoms or dyspnea were observed. Thus, this titanium alloy stent was considered a biocompatible stent.

In this study, the titanium alloy self-expandable stent was found to be easy to insert, and no complications ensued. The tracheal responses to the stent were minimal, without erosion through the tracheal wall and without excessive secretion accumulation. One to 2 weeks after tracheal stenting, all dogs resolved coughing and dyspnea and resumed normal activities. Thus, intratracheal stenting is a less invasive, more effective, straightforward procedure that can be used for end-to-end tracheal anastomosis. In conclusion, it was observed that granular tissue did not invade the tracheal stent and the stent had flexibility and appropriate radial force. These results suggest that a titanium alloy tracheal stent is a helpful device in treating a tracheal anastomotic region during tracheal reconstruction.

\section{Conflicts of Interest}

The Authors declare that they have no competing interests regarding this study.

\section{Authors' Contributions}

This study was designed by Jang and Choi. Lee, Jang and Choi analyzed the diagnostic images and collected the data. All Authors read and approved the final manuscript.

\section{Acknowledgements}

This work was carried out with the support of "Cooperative Research Program for Agriculture Science and Technology Development (Project No. PJ01620801)" Rural Development Administration, Republic of Korea. 


\section{References}

1 Alderson B, Senior JM and Dugdale AH: Tracheal necrosis following tracheal intubation in a dog. J Small Anim Pract 47(12): 754-756, 2006. PMID: 17201831. DOI: 10.1111/j.17485827.2006.00161.x

2 Padrid P and Amis TC: Chronic tracheobronchial disease in the dog. Vet Clin North Am Small Anim Pract 22(5): 1203-1229, 1992. PMID: 1523790 . DOI: $10.1016 / \mathrm{s} 0195-5616(92) 50310-2$

3 White R and Williams J: Tracheal collapse in the dog - is there really a role for surgery? A survey of 100 cases. Journal of Small Animal Practice 35(4): 191-196, 2021. DOI: 10.1111/j.17485827.1994.tb01685.x

4 Tappin SW: Canine tracheal collapse. J Small Anim Pract 57(1): 9-17, 2016. PMID: 26780854. DOI: 10.1111/jsap.12436

5 Raske M, Weisse C, Berent AC, McDougall R and Lamb K: Immediate, short-, and long-term changes in tracheal stent diameter, length, and positioning after placement in dogs with tracheal collapse syndrome. J Vet Intern Med 32(2): 782-791, 2018. PMID: 29460368. DOI: 10.1111/jvim.15063

6 Tinga S, Thieman Mankin KM, Peycke LE and Cohen ND: Comparison of outcome after use of extra-luminal rings and intra-luminal stents for treatment of tracheal collapse in dogs. Vet Surg 44(7): 858-865, 2015. PMID: 26249528. DOI: 10.1111/ vsu. 12365

7 Weisse CW, Berent AC, Todd KL and Solomon JA: Potential applications of interventional radiology in veterinary medicine. J Am Vet Med Assoc 233(10): 1564-1574, 2008. PMID: 19014289. DOI: $10.2460 /$ javma.233.10.1564

8 Lee TK, Kim JM and Choi SH: Pig tracheal patchy xenotransplantation in the dog. Xenotransplantation 26(1): e12452, 2019. PMID: 30117191. DOI: 10.1111/xen.12452

9 Macready DM, Johnson LR and Pollard RE: Fluoroscopic and radiographic evaluation of tracheal collapse in dogs: 62 cases (2001-2006). J Am Vet Med Assoc 230(12): 1870-1876, 2007. PMID: 17571993. DOI: 10.2460/javma.230.12.1870

10 Auchincloss HG and Wright CD: Complications after tracheal resection and reconstruction: prevention and treatment. J Thorac Dis 8(Suppl 2): S160-S167, 2016. PMID: 26981267. DOI: 10.3978/j.issn.2072-1439.2016.01.86

11 Jang SJ, Park MH, Lee TK and Choi SH: Healing effect of platelet-rich plasma on decellularized tracheal allotransplantation in rabbits. In Vivo 32(6): 1443-1447, 2018. PMID: 30348699. DOI: 10.21873 /invivo.11397

12 Dallman MJ, McClure RC and Brown EM: Histochemical study of normal and collapsed tracheas in dogs. Am J Vet Res 49(12): 2117-2125, 1988. PMID: 2467593.

13 Becker WM, Beal M, Stanley BJ and Hauptman JG: Survival after surgery for tracheal collapse and the effect of intrathoracic collapse on survival. Vet Surg 41(4): 501-506, 2012. PMID: 22225498. DOI: 10.1111/j.1532-950X.2011.00945.x
14 Johnson LR and Pollard RE: Tracheal collapse and bronchomalacia in dogs: 58 cases (7/2001-1/2008). J Vet Intern Med 24(2): 298-305, 2010. PMID: 20051001. DOI: 10.1111/ j.1939-1676.2009.0451.x

15 Pink JJ: Intramural tracheal haematoma causing acute respiratory obstruction in a dog. J Small Anim Pract 47(3): 161-164, 2006. PMID: 16512849. DOI: 10.1111/j.1748-5827.2006.00011.x

16 Johnson L: Tracheal collapse. Diagnosis and medical and surgical treatment. Vet Clin North Am Small Anim Pract 30(6): 1253-66, vi, 2000. PMID: 11221980. DOI: 10.1016/s01955616(00)06005-8

17 Argano M, Gendron K, Rytz U and Adami C: Anesthetic and airways management of a dog with severe tracheal collapse during intraluminal stent placement. Case Reports in Veterinary Medicine 2013: 1-5, 2017. DOI: 10.1155/2013/234598

18 Durant AM, Sura P, Rohrbach B and Bohling MW: Use of nitinol stents for end-stage tracheal collapse in dogs. Vet Surg 41(7): 807-817, 2012. PMID: 22957667. DOI: 10.1111/j.1532950X.2012.01037.x

19 Sura PA and Krahwinkel DJ: Self-expanding nitinol stents for the treatment of tracheal collapse in dogs: 12 cases (2001-2004). J Am Vet Med Assoc 232(2): 228-236, 2008. PMID: 18275390. DOI: $10.2460 /$ javma.232.2.228

20 Moritz A, Schneider M and Bauer N: Management of advanced tracheal collapse in dogs using intraluminal self-expanding biliary wallstents. J Vet Intern Med 18(1): 31-42, 2004. PMID: 14765729. DOI: 10.1892/0891-6640(2004)18<31:moatci> 2.0.co;2

21 Violette NP, Weisse C, Berent AC and Lamb KE: Correlations among tracheal dimensions, tracheal stent dimensions, and major complications after endoluminal stenting of tracheal collapse syndrome in dogs. J Vet Intern Med 33(5): 2209-2216, 2019. PMID: 31290188. DOI: 10.1111/jvim.15555

22 Nicholson JW: Titanium alloys for dental implants: A review. Prosthesis 2(2): 100-116, 2020. DOI: 10.3390/prosthesis2020011

23 Young B, Gleeson M and Cripps AW: C-reactive protein: a critical review. Pathology 23(2): 118-124, 1991. PMID: 1720888. DOI: 10.3109/00313029109060809

24 Neumaier M, Metak G and Scherer MA: C-reactive protein as a parameter of surgical trauma: CRP response after different types of surgery in 349 hip fractures. Acta Orthop 77(5): 788-790, 2006. PMID: 17068712 . DOI: $10.1080 / 17453670610013006$

25 Dąbrowski R and Wawron W: Acute-phase response in monitoring postoperative recovery in bitches after ovariohysterectomy. Ann Anim Sci 14: 287-295, 2014. DOI: 10.2478/aoas-2014-0015

Received August 10, 2021

Revised September 1, 2021

Accepted September 2, 2021 